\title{
Os efeitos do método Pilates sobre a força muscular respiratória em crianças com sintomas de asma
}

\author{
The effects of the Pilates method on respiratory muscle strength in children with \\ asthma symptoms
}

\author{
Verônica Benachio Leiria ${ }^{1}$, Simone Lara ${ }^{2 *}$, Susane Graup ${ }^{3}$, Anelise Dumke ${ }^{4}$, Lilian Pinto Teixeira ${ }^{5}$, Patrícia \\ Medeiros Schmidt ${ }^{6}$
}

\begin{abstract}
${ }^{1}$ Acadêmica do Curso de Fisioterapia na Universidade Federal do Pampa (UNIPAMPA), Uruguaiana, RS; ${ }^{2}$ Doutora em Educação e Ciências, Docente do Curso de Graduação de Fisioterapia e de Pós-graduação em Educação em Ciências: Química da Vida e Saúde (UNIPAMPA); ${ }^{3}$ Doutora em Engenharia de Produção, Professora do Curso de Licenciatura em Educação Física e do Programa de Pós-graduação em Educação em Ciências: Química da Vida e Saúde (UNIPAMPA); " Doutora em Ciências Pneumológicas, Professora do Curso de Fisioterapia (UNIPAMPA); ${ }^{5}$ Mestre

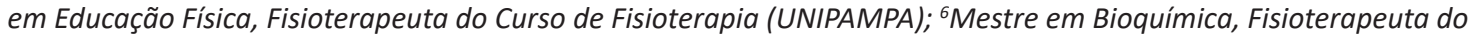
Curso de Fisioterapia (UNIPAMPA)
\end{abstract}

\begin{abstract}
Resumo
Objetivo: analisar os efeitos do método Pilates sobre a força muscular respiratória em crianças com sintomas de asma. Metodologia: nesse estudo clínico foram avaliadas 17 crianças em idade escolar (09 a 12 anos), de ambos os sexos. Inicialmente responderam ao questionário International Study of Asthma and Allergies in Childhood (ISAAC), que avalia a prevalência de asma e doenças alérgicas em crianças e adolescentes. Assim, aquelas que obtiveram uma pontuação no ISAAC, com nota de corte igual ou superior a 5 , foram incluídas no estudo. Após as crianças realizarem uma avaliação através do manovacuômetro, a fim de mensurar as pressões respiratórias máximas (PImáx e PEmáx) foi realizada uma avaliação antropométrica para verificar a massa corporal e a estatura. Como intervenção, as crianças participaram de um programa de exercícios com base no método Pilates 2 vezes por semana, com duração aproximada de 50 minutos cada sessão, durante 8 meses, totalizando 32 semanas. Resultados: Em relação às pressões, tanto a PImáx quanto a PEmáx aumentaram pós-intervenção. Conclusão: o estudo encontrou contribuições significativas do método Pilates sobre a força muscular respiratória (PImáx e PEmáx) de crianças com sintomas de asma. Desta forma, sugere-se que o método representa uma ferramenta importante para melhorar a força muscular respiratória dessa população.

Palavras-chave: Criança. Força muscular. Doenças pulmonares. Fisioterapia.
\end{abstract}

\begin{abstract}
Objective: To analyze the effects of the Pilates method on respiratory muscle function in children with asthma symptoms. Methods: in this clinical study, a sample of school-age children (9 to 12 years old) of both sexes was included. Initially, the children answered the questionnaire International Study of Asthma and Allergies in Childhood (ISAAC). Thus, those who scored 5 or more were included in the study. Subsequent to this initial screening, the children performed an evaluation of respiratory muscle strength through the digital manovacuometer in order to measure the maximum respiratory pressures (MIP and MEP). An anthropometric assessment was also performed to verify body mass and height. The children participated in an exercise program based on the Pilates method, being performed twice a week, lasting about 50 minutes each session, for 8 months, totaling 32 weeks. Results: regarding the pressures, both inspiratory and expiratory pressure increased at the end of the intervention. Conclusion: the present study found significant contributions of the Pilates method in the inspiratory muscle strength of children with asthma symptoms. Thus, it is suggested that the method represents an important tool to improve the muscular strength of these children.

Keywords: Child. Muscle strength. Pulmonary diseases. Physiotherapy.
\end{abstract}

\section{INTRODUÇÃo}

A asma representa uma doença crônica e inflamatória, caracterizada por hiperresponsividade das vias aéreas e por limitação variável ao fluxo aéreo, sendo resultado de uma interação entre genética, alérgenos, fumaça de cigarro ou outros fatores que possam levar ao desenvolvimento e manutenção dos sintomas ${ }^{1,2}$. Manifesta-se

Correspondente/Corresponding: *Simone Lara - End: UNIPAMPA, Campus Uruguaiana/RS, BR 472, KM 592, 97508-000, Brasil, CX Postal 118. - Tel: (55) 39110200. - E-mail: slarafisio@yahoo.com.br clinicamente por episódios recorrentes de dispneia, sibilância, dor torácica e tosse, variando de intensidade e ao longo do tempo ${ }^{2}$.

Uma revisão sistemática com meta-análise encontrou que fatores como sexo masculino, exposição à fumaça, histórico familiar de asma, história de sibilos, dentre outros, estiveram significativamente associados ao desenvolvimento de asma na pré-escola ou na idade escolar precoce ${ }^{3}$, sendo que a prevalência e a gravidade da asma têm aumentado consideravelmente nas últimas décadas ${ }^{4}$. 
Barreto et $a l .{ }^{5}$ identificaram uma alta prevalência de sintomas $(23,2 \%)$ e de relato de diagnóstico médico prévio de asma $(12,4 \%)$ em uma amostra de adolescentes brasileiros, e concluíram que o Brasil está entre os países com maior prevalência de asma no mundo e a prevalência ainda é crescente. Corroborando, em um estudo envolvendo escolares brasileiros, a prevalência total de asma ativa foi de $13,1 \%$, sendo uma taxa considerada alta ${ }^{6}$.

Ainda que autores apontem efeitos positivos da prática regular de exercícios físicos em crianças asmáticas, como a redução de dispneia, do consumo de corticosteroides inalatórios, do número de exacerbações e a intensidade da broncoconstrição ${ }^{7}$, existe a necessidade de ampliar os estudos sobre os possíveis efeitos da prática regular de atividades físicas para o controle da asma nessa faixa etária ${ }^{8}$.

Nesse contexto, o método Pilates representa um método de condicionamento físico e mental prazeroso, que é aplicado a qualquer idade respeitando os limites de cada indivíduo ${ }^{9}$, incluindo a respiração, a concentração, o controle, a centralização, a precisão e a fluidez como princípios fundamentais, os quais são associados ao baixo impacto na execução dos movimentos ${ }^{10}$. A prática desse método oferece inúmeros benefícios, dentre eles uma boa execução das funções respiratórias, onde revela um impacto significante na alteração da capacidade ventilatória e do desempenho da respectiva musculatura ${ }^{11}$. Da mesma forma que o método Pilates melhora a função muscular respiratória em adultos saudáveis ${ }^{12}$, bem como em idosos saudáveis ${ }^{13}$, supomos que a população infantil também possa ser beneficiada.

Contudo, devido à escassez de trabalhos que reportem a influência desse método sobre a força respiratória na infância, sobretudo em crianças com sintomas de asma, o objetivo do presente estudo foi analisar os efeitos do método Pilates sobre a força muscular respiratória em crianças com sintomas de asma.

\section{METODOLOGIA}

\section{Estudo e amostra}

O desenho metodológico da pesquisa é uma série de casos, com caráter descritivo e quantitativo, no qual foi incluída uma amostra formada por 17 crianças com idades entre 09 e 12 anos, de ambos os sexos, estudantes dos 4 ㅇ e 5음 anos do turno da tarde, de uma escola pública, localizada na periferia de um município da fronteira oeste do estado do Rio Grande do Sul, Brasil. A escolha da referida escola se deu pelo fato de a mesma possuir um Estúdio de Pilates montado em suas instalações, sendo o mesmo de propriedade de uma Universidade Federal que utiliza o espaço para projetos de pesquisa, ensino e extensão com a comunidade.

Inicialmente, as crianças foram selecionadas por meio de uma triagem inicial utilizando o questionário ISAAC - "International Study of Asthma and Allergies in Childhood", que foi criado para avaliar a prevalência de asma e doenças alérgicas em crianças e adolescentes, sendo autoaplicável e de fácil entendimento, sendo composto por oito perguntas sobre a presença de sinais e sintomas de asma e uma pergunta referente ao diagnóstico de asma, na segunda fase ${ }^{14}$. Após a aplicação do questionário, foram incluídas no estudo, as crianças que apresentaram uma pontuação mínima de 5 pontos, critério esse também utilizado no estudo de Wandalsen et al. ${ }^{15}$.

Assim, os critérios de inclusão do estudo foram: crianças de ambos os sexos, que cursavam o quarto e quinto ano do ensino fundamental e que apresentassem sintomas de asma, por meio de triagem prévia por meio do questionário ISAAC. Foram excluídas as crianças com diagnóstico clínico de qualquer patologia ortopédica e/ ou neurológica, atestada por meio de laudo médico. Cabe ressaltar que todos os preceitos éticos foram respeitados, no qual os responsáveis legais pelo estudante assinaram o termo de consentimento livre e esclarecido (TCLE), e as crianças assinaram o termo de assentimento. $O$ estudo foi aprovado no Comitê de Ética e Pesquisa institucional (registro 2.063.873).

\section{Instrumentos de avaliação}

Para a avaliação da força muscular respiratória das crianças foi utilizado um manovacuômetro digital, sendo um método simples, rápido e não invasivo, que consiste na mensuração das pressões respiratórias máximas (PImáx e PEmáx), que indicam, respectivamente, a força da musculatura inspiratória e expiratória contra uma via aérea ocluída ${ }^{16}$. A pressão inspiratória máxima (PImáx) foi obtida por meio de uma manobra de esforço inspiratório máximo após a realização de uma expiração máxima, próxima ao volume residual (VR) e a pressão expiratória máxima (PEmáx) foi obtida por meio de um esforço expiratório máximo, após uma inspiração máxima, próxima a capacidade pulmonar total (CPT) ${ }^{17}$.

Estas mensurações foram realizadas com a criança em sedestação, com o tronco ereto em uma posição de 90 graus de quadril e com a utilização de um clipe nasal para evitar o escape de ar pelo nariz. O encorajamento verbal foi fornecido em todas as medições e para evitar vazamento e acúmulo de ar na região lateral da cavidade oral, a criança foi instruída a segurar com as mãos a musculatura da face durante a avaliação da PEmáx. As manobras foram realizadas, no mínimo três vezes e no máximo cinco vezes, sendo que o último valor registrado não poderia ser maior que os anteriores ${ }^{18}$. As pressões respiratórias foram classificadas de acordo com os valores propostos por Domènech-Clar et al. ${ }^{19}$, que considera o sexo e a idade com referências.

Ainda, foi calculada a PImáx e a PEmáx predita para meninas e meninos nos momentos pré e pós intervenção e comparadas com os valores apresentados nas avaliações ${ }^{20}$. As equações estão expressas a seguir, sendo que com base nas mesmas será calculado o percentual atingido pelo avaliado. 
PImáx predita para meninas $=14,226-(0,551 \times$ estatura) $-(0,638 \times$ massa $)$

PImáx predita para meninos $=17,879-(0,674 \times$ estatura) - (0,604 $\times$ massa)

PEmáx predita para meninas $=30,046+(0,749 x$ estatura $)+(4,213+$ idade $)$

PEmáx predita para meninos $=47,417+(0,898 \times$ estatura $)+(3,166+$ idade $)$

$\mathrm{Na}$ avaliação antropométrica, a massa corporal foi verificada por meio de uma balança antropométrica, na qual o indivíduo deveria estar com roupas leves e descalço. A estatura foi verificada em um estadiômetro fixo na parede, no qual a criança deveria estar em posição ereta e descalça. Com base nestas avaliações, foi realizado o cálculo para a definição do Índice de Massa Corporal IMC (massa corporal/estatura²) e para a classificação foi utilizada a tabela do Projeto Esporte Brasil (PROESP-Br) $)^{21 .}$

\section{Protocolo de intervenção}

As crianças participaram de um programa de exercícios com base no método Pilates, supervisionadas pelos pesquisadores, os quais realizaram treinamento prévio para padronização da linha de formação do método. Os exercícios foram executados na escola, sendo duas vezes por semana, com duração aproximada de 50 minutos cada sessão, durante 8 meses, totalizando 32 semanas. As crianças foram subdivididas em pequenos grupos, de forma que cada uma tivesse orientação de um pesquisador durante as práticas, para possíveis correções durante a prática dos exercícios. Os exercícios foram realizados no solo, com acessórios e com aparelhos do método, envolvendo 01 série de 08 repetições cada, e subdivididos em três protocolos, evoluindo conforme grau de dificuldade, assim sendo: Protocolo 01: Desenvolvido nas primeiras 10 semanas, envolvendo exercícios básicos do método. Protocolo 02: Desenvolvido entre as próximas 10 semanas, incluindo exercícios intermediários e o Protocolo 03: Desenvolvidas nas últimas 12 semanas, (quadro 1 ).

Quadro 1 - Protocolo de exercícios do método Pilates

\begin{tabular}{|l|l|l|}
\hline Protocolo 1 & Protocolo2 & Protocolo 3 \\
\hline $\begin{array}{l}\text { Preparação da } \\
\text { respiração }\end{array}$ & $\begin{array}{l}\text { Preparação da } \\
\text { respiração }\end{array}$ & $\begin{array}{l}\text { Preparação da } \\
\text { respiração }\end{array}$ \\
\hline Cat (Solo) & Solo legpull front & Legpull front (Solo) \\
\hline $\begin{array}{l}\text { Spinestretch } \\
\text { (Trapézio) }\end{array}$ & $\begin{array}{l}\text { BodyExtension } \\
\text { (Meia Lua) }\end{array}$ & Rollup (Solo) \\
\hline $\begin{array}{l}\text { Rolling back } \\
\text { (Trapézio) }\end{array}$ & $\begin{array}{l}\text { Body up and down } \\
\text { (MeiaLua) }\end{array}$ & Double leg kick \\
\hline $\begin{array}{l}\text { Leglowers + legcircle } \\
\text { (Reformer) }\end{array}$ & $\begin{array}{l}\text { Side body twist } \\
\text { (Barrel) }\end{array}$ & Pinwhell (Solo) \\
\hline $\begin{array}{l}\text { Arms: upanddown } \\
\text { (Reformer) }\end{array}$ & Tower (Trapézio) & Swan front (Chair) \\
\hline $\begin{array}{l}\text { Going up and front } \\
\text { (Chair) }\end{array}$ & Beathing (Trapézio) & Ponte unipodal (Chair) \\
\hline $\begin{array}{l}\text { Pumponelegside } \\
\text { (Chair) }\end{array}$ & Swan front (Chair) & Ponte (Reformer) \\
\hline
\end{tabular}

\begin{tabular}{|l|l|l|}
\hline Situp (Barrel) & $\begin{array}{l}\text { Ponte unipodal } \\
\text { (Chair) }\end{array}$ & $\begin{array}{l}\text { KneestrechesRound } \\
\text { (Reformer) }\end{array}$ \\
\hline Spine Twist (Barrel) & $\begin{array}{l}\text { Front slips } \\
\text { (Reformer) }\end{array}$ & $\begin{array}{l}\text { Adução horizontal do } \\
\text { ombro (Trapézio) }\end{array}$ \\
\hline Ponte (Bola Suiça) & Sideslips (Reformer) & $\begin{array}{l}\text { Abdução horizontal } \\
\text { do ombro (Trapézio) }\end{array}$ \\
\hline Swan (Bola Suiça) & & \\
\hline
\end{tabular}

Fonte: Autoria própria

\section{Analise estatística}

Para a análise dos dados foi utilizada estatística descritiva com média, desvio padrão, frequências relativas. A normalidade dos dados foi testada pelo teste de Shapiro-Wilk que identificou uma distribuição normal. A diferença entre as avaliações foi testada pelo Teste " $t$ " de Student para amostras dependentes. Os cálculos foram realizados por meio do software Statistical Package for the Social Sciences (SPSS - versão 20.0), e um nível de significância de $5 \%$ foi utilizado em todos os procedimentos estatísticos realizados no estudo.

\section{RESULTADOS}

Foram avaliadas 17 crianças com idade média de 9,6 $\pm 0,93$ anos, sendo $9(52,9 \%)$ do sexo masculino. Na tabela 1 , verificamos que as crianças aumentaram a massa corporal e a estatura após a intervenção $(p<0,01)$. Em relação às pressões, tanto os valores médios das pressões inspiratória quanto a expiratória aumentaram ao final da intervenção $(p<0,01)$. Ainda na tabela 1 , foi possível verificar que, a diferença entre a PEmáx predita e a realizada não teve alteração significativa com a intervenção $(p=$ $0,128)$. Por outro lado, a diferença entre a PImáx predita e a realizada diminuiu com a intervenção $(p<0,001)$.

Tabela 1 - Perfil antropométrico e força muscular respiratória antes e após a intervenção IMC: índice de massa corporal, PImáx: Pressão inspiratória máxima, PEmáx: Pressão expiratória máxima.

\begin{tabular}{|c|c|c|c|}
\hline \multirow{2}{*}{ Variável } & Pré-intervenção & Pós-intervenção & \multirow[t]{2}{*}{$\mathbf{P}$} \\
\hline & Média \pm DP & Média \pm DP & \\
\hline Massa Corporal (kg) & $46,7 \pm 19,41$ & $49,7 \pm 21,28$ & $<0,001 *$ \\
\hline Estatura $(\mathrm{m})$ & $1,43 \pm 0,09$ & $1,46 \pm 0,102$ & $<0,001 *$ \\
\hline $\mathrm{IMC}\left(\mathrm{kg} / \mathrm{m}^{2)}\right.$ & $22,5 \pm 7,49$ & $22,7 \pm 7,78$ & 0,240 \\
\hline PImáx $\left(\mathrm{cmH}_{2} \mathrm{O}\right)$ & $-80,1 \pm 21,93$ & $-103,8 \pm 30,23$ & $0,001 *$ \\
\hline PEmáx $\left(\mathrm{cmH}_{2} \mathrm{O}\right)$ & $91,4 \pm 35,17$ & $106,2 \pm 28,64$ & $0,046^{*}$ \\
\hline PImáx predita $\left(\mathrm{cmH}_{2} \mathrm{O}\right)$ & $-100,66 \pm 15,02$ & $-105,46 \pm 16,68$ & $<0,001 *$ \\
\hline PEmáx predita $\left(\mathrm{cmH}_{2} \mathrm{O}\right)$ & $170,47 \pm 18,26$ & $173,35 \pm 18,43$ & $<0,001 *$ \\
\hline $\begin{array}{l}\Delta \text { entre PImáx predita e } \\
\text { realizada }\end{array}$ & $-20,6 \pm 28,33$ & $-1,64 \pm 36,75$ & $<0,001 *$ \\
\hline $\begin{array}{l}\Delta \text { entre PEmáx predita e } \\
\text { realizada }\end{array}$ & $79,12 \pm 40,06$ & $67,12 \pm 36,83$ & 0,128 \\
\hline $\begin{array}{l}\% \text { de PImáx atingido a } \\
\text { partir do previsto }\end{array}$ & $81,2 \pm 25,8$ & $100,7 \pm 32,0$ & $<0,001 *$ \\
\hline $\begin{array}{l}\% \text { de PEmáx atingido a } \\
\text { partir do previsto }\end{array}$ & $54,1 \pm 21,3$ & $62,2 \pm 18,4$ & 0,128 \\
\hline
\end{tabular}

Fonte: Dados da pesquisa 
Considerando a classificação das pressões, a Figura 1 mostra o percentual de crianças que atingiram a equação prevista para a PImáx e para PEmáx, sendo possível verificar que após a intervenção $100 \%$ das crianças atingiram os valores previstos para a PEmáx.

Figura 1 - Percentual de crianças que atingiram as pressões previstas pelas equações.

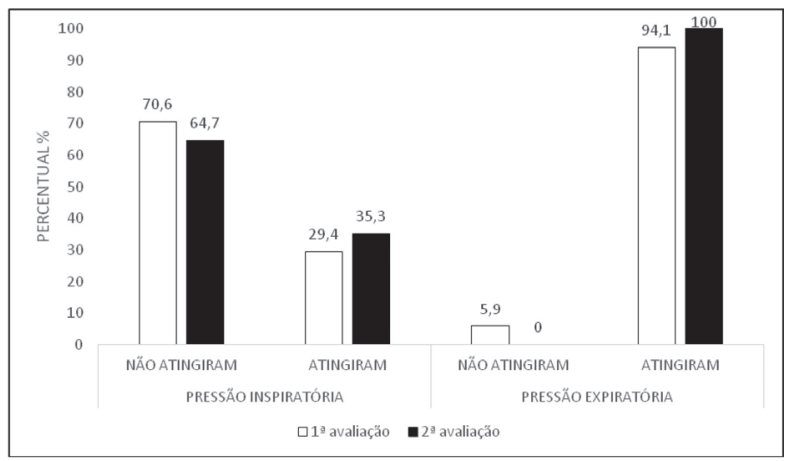

Fonte: Autoria própria

Por outro lado, em relação à classificação dos valores brutos das pressões expressas pelas crianças foi possível verificar que $100 \%$ apresentaram valores de PImáx considerados normais após a intervenção.

Figura 2 - Distribuição de frequência da classificação das pressões inspiratória (PImax) e expiratória (PEmax) das crianças avaliadas.

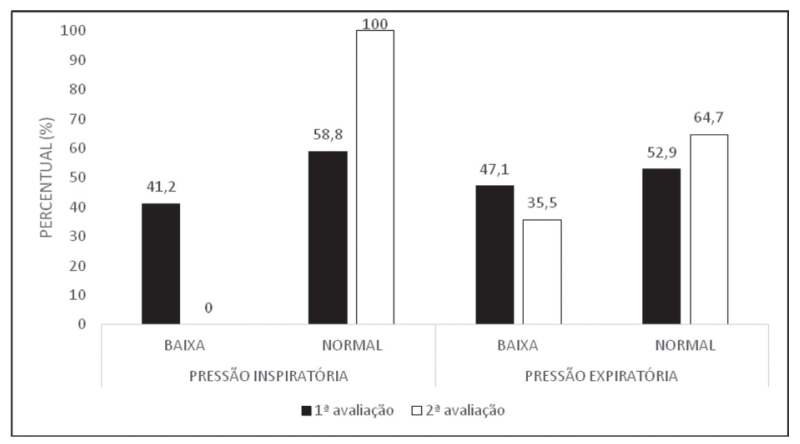

Fonte: Autoria própria

\section{DISCUSSÃO}

O presente estudo identificou um aumento nos valores de força muscular inspiratória e expiratória em crianças, após a prática do método Pilates. Tendo em vista que os sujeitos apresentaram aumento na estatura e na massa corporal, também realizamos uma análise buscando comparar a diferença entre as pressões preditas e executadas, e evidenciamos uma melhora na PImáx pós-intervenção. Indo ao encontro desse achado, identificamos que $100 \%$ das crianças apresentaram valores de PImáx considerados normais após a intervenção, bem como $100 \%$ das crianças atingiram os valores previstos para a PEmáx pós-intervenção.
Esses resultados são relevantes pois demonstram que o método pode representar uma estratégia eficaz para promover o desenvolvimento da força muscular respiratória em crianças com sintomas de asma. Apesar das crianças do presente estudo não apresentarem um diagnóstico clínico de asma, as mesmas exibiram sinais importantes de disfunção respiratória, uma vez que apenas $58,8 \%$ delas apresentou a PImáx normal, e $52,9 \%$ a PEmáx normal, pré-intervenção.

Sugere-se que tais melhorias encontradas nesse trabalho ocorreram devido aos próprios princípios propostos pelo método, ou seja, a centralização e a respiração. No princípio da centralização, enfatiza-se a contração isométrica dos músculos abdominais durante todos os exercícios do método ${ }^{9}$. Além disso, tal princípio faz com que o principal músculo inspiratório, o diafragma, encontre-se em posição de alongamento, e, assim, os músculos que auxiliam a inspiração também se tornam fortalecidos, contribuindo para o aumento da força muscular inspiratória ${ }^{22}$. Jesus et al..$^{23}$ sugerem que esse princípio, norteador do método Pilates, possibilita o recrutamento dos músculos do tronco e da parede abdominal, assim como do diafragma, melhorando assim, a força dos músculos respiratórios.

Quanto ao princípio da respiração, Barbosa et al. ${ }^{24}$ compararam a ativação do músculo transverso do abdome durante a flexão do tronco com e sem a técnica de respiração do Pilates, e encontraram uma maior ativação muscular naqueles indivíduos que utilizaram a respiração do método. Os autores sugerem que a utilização dessa respiração possa contribuir também no processo de ativação muscular e, conseqüentemente, melhorar a função muscular respiratória dos indivíduos.

Indo ao encontro desses achados Barbosa et al. ${ }^{25}$ comparam a ativação dos músculos estabilizadores centrais e músculos abdominais em 23 indivíduos com e sem experiência em Pilates, sendo que o grupo experiente deveria ter no mínimo três meses de prática do método, e o grupo sem experiência nunca experimentou o mesmo. Os autores encontraram que os indivíduos que praticaram o método Pilates foram capazes de sustentar a contração muscular abdominal e lombar profunda concomitantemente durante a manobra de contração do transverso do abdome, enquanto que os indivíduos sem experiência não conseguiram atingir os mesmos níveis de ativação abdominal e não apresentaram co-ativação significativa da musculatura profunda lombar durante a manobra solicitada. Com base nos resultados desse estudo, acredita-se que a prática do método Pilates melhore a ativação dos músculos estabilizadores centrais e abdominais, o que corrobora com os dados do presente estudo.

Estudos envolvendo os efeitos desse método sobre a força muscular respiratória são conhecidos em outras populações, como em mulheres saudáveis ${ }^{26} \mathrm{e} \mathrm{em}$ idosas ${ }^{27}$. Nesse sentido, Giacomini et al. ${ }^{26}$, em seu ensaio clínico não controlado, buscaram identificar os efeitos do método Pilates sobre a função muscular respiratória em 16 muIheres saudáveis e sedentárias. Os autores encontraram 
que houve contribuições da prática do método sobre a força e o desempenho muscular respiratório, bem como sobre espessura muscular da parede abdominal pós-intervenção. Alvarenga et $a .^{27}$ identificaram melhorias sobre a função muscular (PImáx e PEmáx) em 36 idosas, após a prática de um treinamento muscular inspiratório combinado com o método Pilates.

Contudo, essa forma de intervenção na infância ainda é limitada, como evidenciou a revisão sistemática proposta por Hornsby e Johnston ${ }^{28}$. Esse trabalho foi a primeira revisão a ser realizada para identificar os efeitos do método voltado ao público infanto-juvenil, e os autores encontraram que o método proporcionou melhorias sobre a flexibilidade, força muscular e controle postural, além de reduzir a dor em crianças com patologia musculoesquelética. Entretanto, estes autores sugerem que mais pesquisas são necessárias para determinar o potencial efeito do Pilates em crianças e jovens para vários grupos populacionais. Ademais, Jesus et al. ${ }^{23}$ recomendam que novos estudos sejam realizados em populações com disfunções respiratórias, já que há uma lacuna de trabalhos nesse viés. Esses autores reforçam a importância do nosso estudo, em que analisamos os efeitos do método Pilates no público infantil e com sintomas de asma.

De forma semelhante aos nossos achados, Montes ${ }^{29}$ analisou os efeitos do método em 21 adultos jovens com asma controlada, e os resultados evidenciaram que a prática contribuiu para a ativação dos músculos eretor da coluna, multífidos, transverso/oblíquo interno e do diafragma, bem como sobre a pressão expiratória máxima. Já o estudo de Franco et al. ${ }^{30}$ analisaram os efeitos de 16 semanas de prática do método Pilates, em 19 pacientes jovens (média de 13 anos de idade) portadores de fibrose cística. Os autores encontraram um aumento significativo da PImáx nos pacientes do sexo masculino, enquanto houve aumentos significativos na PImáx e PEmáx nos pacientes do sexo feminino, após a intervenção.

Considerando que esses estudos evidenciaram efeitos positivos da prática do Pilates em pacientes com disfunções respiratórias (sejam adolescentes ou adultos), sugere-se que tais efeitos possam ser estendidos ao público infantil, como percebemos em nosso estudo. Porém, devido à lacuna existente nesta área, acreditamos ser fundamental a realização de mais trabalhos reportando os efeitos dessa intervenção em crianças com disfunções respiratórias.

Como limitações do estudo destacam-se a não realização do exame de espirometria para diagnosticar com mais precisão a asma dessas crianças, bem como a ausência de um grupo controle, a fim de obter comparações mais conclusivas.

\section{CONCLUSÃO}

O presente estudo encontrou contribuições significativas do método Pilates sobre a força muscular respiratória (PImáx e PEmáx) de crianças com sintomas de asma.
Por meio da classificação das pressões, identificamos que $100 \%$ das crianças apresentaram valores de PImáx considerados normais após a intervenção. Ademais, evidenciamos que $100 \%$ das crianças atingiram os valores previstos para a PEmáx após a intervenção.

Ainda, destacamos a necessidade da realização de mais trabalhos, envolvendo a prática deste método em indivíduos com disfunções respiratórias, como por exemplo, a asma, bem como voltados à população infantil, já que os estudos mais próximos incluem idosos e mulheres saudáveis.

\section{REFERÊNCIAS}

1. REDDEL, H.K. et al. A summary of the new GINA strategy: a roadmap to asthma control. Eur. respir. j., Copenhagen, v.46, n.3, p.622-39, 2015.

2. ASMA SBdPeTpoMd. Diretrizes da Sociedade Brasileira de Pneumologia e Tisiologia para o Manejo da Asma. J. Bras. Pneumol., Brasilia,v.38, n.1, p.44-58, 2012.

3. BAO, Y. et al. Risk Factors in Preschool Children for Predicting Asthma During the Preschool Age and the Early School Age: a Systematic Review and Meta-Analysis. Curr. allergy asthma rep., Philadelphia, v.17, n.12, p.85, 2017

4. den DEKKER, H.T. et al. Newborn DNA-methylation, childhood lung function, and the risks of asthma and COPD across the life course. Eur. respir. J., Copenhagen, v.53, n.4, 1801795, 2019.

5. BARRETO, M.L. et al. Prevalence of asthma symptoms among adolescents in Brazil: National Adolescent School-based Health Survey (PeNSE 2012). Rev. bras. epidemiol., São Paulo, v.17, p.106-15, 2014.

6. KUSCHNIR, F.C. et al. ERICA: prevalence of asthma in Brazilian adolescents. Rev. saúde pública, São Paulo, v.50, supl. 1, p. 13s, 2016.

7. SOUSA, A.W. et al. Barriers to daily life physical activities for Brazilian children with asthma: a cross-sectional study. J.asthma., New York, v.57, n.6, p.1-9, 2019.

8. SANTOS, A.P. dos, et al. Efeito da atividade física no controle da asma em escolares. Einstein (São Paulo), São Paulo, v.18,n. eAO4936, 2020

9. NIEHUES, J.R. et al. Pilates Method for Lung Function and Functional Capacity in Obese Adults. Altern. ther. health med., Aliso Viejo, v. 21, n.5, p.73-80, 2015.

10. LIBERALINO, E.S.T.; SOUSA, T.C.C.; SILVA, V.R.L. Influência dos exercícios do método Pilates sobre o sistema cardiorrespiratório. REBES, Pombal, v.3, n.3, p.59-66, 2013.

11. BARBOSA, J.D.M.I.; BARROS, D.M.F.P.; GARDENGHI, G. A influência do método Pilates na força muscular respiratória - Revisão de literatura. Revista Eletronica Saúde e Ciência, [s.I], v.6, n.2, p.36-11, 2016.

12. SANTOS, N.T.O. et al. Desempenho muscular respiratório após 12 sessões de treinamento utilizando o aparelho Reformer do método Pilates. Fisioter. Pesqui., São Paulo, v. 26, p.58-64, 2019.

13. LOPES, E.D.S.; RUAS, G.; PATRIZZI, L.J. Efeitos de exercícios do método Pilates na força muscular respiratória de idosas: um ensaio clínico. Rev. bras. geriatr. gerontol., Rio de Janeiro, v.17, p.517-23, 2014.

14. SOLÉ, D. et al. Asthma in children and adolescents in Brazil: contribution of the International Study of Asthma and Allergies in Childhood (ISAAC). Rev. paul. pediatr., São Paulo, v.32, p.114-125, 2014.

15. WANDALSEN, N.F. et al. Avaliação de critérios para o diagnóstico de 
asma através de um questionário epidemiológico. J. Bras. Pneumol., Brasilia, v.35, n.3, p.199-205, 2009.

16. SANDI, N.E.F.; SILVA, L.D. Análise comparativa da força dos músculos respiratórios em indivíduos saudáveis no solo e na piscina. Fisioter. Pesqui., São Paulo, v.25, p.182-187, 2018.

17. BESSA, E.J.C.; LOPES, A.J.; RUFINO, R. A importância da medida da força muscular respiratória na prática da pneumologia. Revista Pulmão, Rio de Janeiro, v.24, n.1, p.37-35, 2015.

18. PEREIRA, N.H. et al. Comparação dos valores obtidos e previstos das pressões respiratórias máximas em adultos jovens. Ciênc. cuid. saúde., Maringá, v.14, n.1, p. 955-957, 2015.

19. DOMÈNECH-CLAR, R. et al. Maximal static respiratory pressures in children and adolescents. Pediatr Pulmonol., New York, v.35, n.2, p.126-32, 2003.

20. HEINZMANN-FILHO J.P. et al. Normal values for respiratory muscle strength in healthy preschoolers and school children. Respir. med., London, v.106, n.12, p.1639-1646, 2012.

21. GAYA, A.; GAYA, A. Projeto esporte Brasil PROESP-Br. Manual de testes e avaliação. Disponível em: http://www.proe,sp.ufrgs.br2016. Acesso em: 05 out. 2019.

22. TORRI, B.G. et al. O Método Pilates melhora a função pulmonar e a mobilidade torácica de pacientes com doença pulmonar obstrutiva crônica. Fisioter Bras., Rio de Janeiro, v.18, n.1, p.56-62, 2017.

23. JESUS, L.T. et al. Efeitos do método Pilates sobre a função pulmonar, a mobilidade toracoabdominal e a força muscular respiratória: ensaio clínico não randomizado, placebo-controlado. Fisioter. Pesq., São Paulo, v.22, p.213-22, 2015.

24. BARBOSA, A.W. et al. The Pilates breathing technique increases the electromyographic amplitude level of the deep abdominal muscles in untrained people. J. bodyw. mov. ther., New York, v.19, n.1, p.57-61, 2015.

25. BARBOSA, A.C. et al. Pilates experience vs. muscle activation during abdominal drawing-in maneuver. J. bodyw. mov. ther., New York, v.22, n.2, p.467-70,2018.

26. GIACOMINI, M.B. et al. The Pilates Method increases respiratory muscle strength and performance as well as abdominal muscle thickness. J. bodyw. mov. ther., New York, v.20, n.2, p.258-64, 2016.

27. ALVARENGA, G.M. et al. The influence of inspiratory muscle training combined with the Pilates method on lung function in elderly women: A randomized controlled trial. Clinics., São Paulo, v.73, 2018.

28. HORNSBY, E.; JOHNSTON, L.M. Effect of Pilates intervention on physical function of children and youth: A systematic review. Arch. phys. med. rehabil., Chicago, v.101, n.2, p. 317-328, 2019.

29. MONTES, A. Efeito de um programa de exercícios segundo Pilates em indivíduos com asma controlada. controlo motor vs. função ventilatória. (Dissertação de Mestrado), Instituto Politécnico do Porto. Escola Superior de Tecnologia da Saúde do Porto, Portugal, 2011.

30. FRANCO, C.B. et al. Effects of Pilates mat exercises on muscle strength and on pulmonary function in patients with cystic fibrosis. J. bras. pneumol., Brasilia, v.40, p.521-7, 2014.

Submetido em: $11 / 08 / 2020$

Aceito em: 17/11/2020 